\title{
ANÁLISIS DE LAS SITUACIONES DE VIOLENCIA ENTRE IGUALES EN EDUCACIÓN PRIMARIA: DIFERENCIAS EN FUNCIÓN DEL GÉNERO Y DEL CURSO
}

\author{
Beatriz Lucas Molina \\ Universidad de La Rioja \\ Rosa Pulido Valero \\ Universidad Complutense de Madrid \\ Gema Martín Seoane \\ Universidad Complutense de Madrid
}

\begin{abstract}
RESUMEN: El objetivo del presente estudio fue conocer la relación entre las conductas de violencia entre iguales, el género y el curso, en Educación Primaria. Los participantes fueron 2.050 escolares de 8 a 13 años de 27 centros públicos y privado-concertados de Educación Primaria de la Comunidad de Madrid. La muestra fue diseñada para que representase a todo el alumnado escolarizado entre $3^{\circ}$ y $6^{-}$curso de la región. El alumnado cumplimentó un cuestionario sobre distintas situaciones de violencia entre iguales. Los análisis factoriales revelaron la existencia de dos dimensiones: 'violencia física y ataques a la propiedad' y 'violencia verbal y exclusión social'. Los chicos informaron sufrir y ejercer con mayor frecuencia que las chicas tanto la violencia física como la verbal-exclusión social. No hubo efecto del curso en las diferencias de género encontradas. Este trabajo nos proporciona una mejor comprensión de la violencia entre iguales en Educación Primaria, así como posibles orientaciones para su prevención.
\end{abstract}

ABSTRACT: The aim of the present study was to study the relationship among gender, school grade and peer harassment at Primary School. The participants were 2.050 children aged 8 to 13 . The overall sample was designed to represent all students in grades $3^{\text {th }}$ through $6^{\text {th }}$ in both public and private schools. A self-report questionnaire on peer harassment situations was administered to the participants. Factor analysis revealed two different dimensions: 'physical violence and property attacks' and 'verbal violence and social exclusion'. Boys reported higher levels of peer harassment among classmates than girls. No effect of the school grade on the gender differences 
was found. This paper provides a better understanding of peer harassment as well as some prevention indications.

PALABRAS CLAVE: Violencia entre iguales, género, curso, Educación Primaria, prevención.

KEYWORDS: Peer harassment, gender, school grade, Primary School, prevention.

\section{MARCO TEÓRICO}

Los resultados de las investigaciones realizadas dentro (Ararteko, 2006; Defensor del Menor, 2006; Defensor del Pueblo, 2000, 2006; Díaz-Aguado, Martínez Arias y Martín Seoane, 2004; Garaigordobil y Oñederra, 2009; Ortega y Mora-Merchán, 2000; Síndic de Greuges, 2007) y fuera de nuestro país (Espelage, Holt y Henkel, 2003; Currie et al., 2004; Nansel, et al., 2004; Olweus, 1993; Owens, Dali y Slee, 2005, 2005; Pellegrini, 2002; Veenstra, Lindenberg, Munniksma y Dijkstra, 2010) sobre el acoso y la violencia entre escolares, señalan tendencias generales que apuntan a claras diferencias de género tanto en el grado de participación como en la severidad de estos fenómenos.

Desde el primer trabajo realizado al respecto (Olweus, 1978) hasta los más recientes (Currie et al., 2004; Nansel et al., 2004; Veenstra et al., 2010) se ha puesto de manifiesto que los chicos informan ejercer y sufrir mayor número de situaciones de violencia por parte de sus iguales que sus compañeras. Y esta diferencia no es sólo cuantitativa, sino también cualitativa: los chicos se involucran en episodios de violencia que revisten mayor gravedad que los protagonizados por las chicas.

Estas diferencias en la participación y gravedad de las conductas de violencia entre iguales tienen su antecedente en los estudios realizados sobre la agresión en los que se concluía que los chicos eran mucho más agresivos que las chicas (Block, 1983; Parke y Slaby, 1983).

Sin embargo, a partir de mediados de los 90, Nicki Crick y Jennifer Grotpeter (1995) empezaron a sospechar que tal vez estas diferencias de género no eran debidas a una mayor tendencia a la agresión por parte de los chicos, sino a que las medidas empleadas estaban sesgadas y sólo tenían en cuenta aquellas formas de agresión directas (como la agresión física), más visibles y mucho más comunes entre los chicos. Estas autoras defendían que las pautas de agresión de las chicas eran distintas a las de los chicos. De este modo, mientras que los chicos utilizaban de forma general la agresión física y verbal para hacer daño a los demás porque este modo de proceder era consistente con el objetivo de dominio físico existente entre los grupos de chicos (Block, 1983); las chicas, por su parte, tenderían a usar la agresión relacional con sus compañeras (Crick y Grotpeter, 1995). Este tipo de comportamiento agresivo sería consistente con los objetivos de intimidad y de pertenencia presentes entre los grupos de chicas, e incluiría conductas como la exclusión del grupo de juego como medida de represalia, la retirada intencional de la amistad como forma de herir y ejercer el poder, y la difusión de rumores para fomentar el rechazo grupal hacia una determinada niña (Crick y Grotpeter, 1995; Crick, Nelson, Morales, Cullerton-Sen, y Hickman, 2001). Así, estas autoras iniciaron la inclusión de este tipo de agresión, 
mucho menos visible, considerado como típicamente femenino y dirigido a dañar las relaciones de amistad o el sentimiento de pertenencia a un grupo, en sus trabajos de investigación sobre el tema.

Cuando se han incluido ambas formas de agresión o acoso, se ha visto, de forma general, que los chicos tienen mayor tendencia a involucrarse en situaciones de acoso físico que las chicas (Espelage, Bosworth y Simon, 2000; Garaigordobil y Oñederra, 2009; Lucas Molina, 2008; Olweus, 1999; Pellegrini y Long, 2002; Smith y Sharp, 1994; Sullivan, 2000; Salmivalli, 2010). Sin embargo, las diferencias en el acoso verbal son menos consistentes (Knight, Gutherie, Page y Fabes, 2002; Martín Seoane, Pulido y Vera, 2008). Las chicas emplean en mayor medida la violencia relacional o indirecta que los chicos (Crick, 1996; Crick et al., 2001; Crick y Grotpeter, 1995), aunque algunos estudios no encuentran diferencias en este sentido (Nansel et al., 2001; Prinstein, Boergers y Vernberg, 2001; Tapper y Boulton, 2004; Toldos, 2005).

Es importante señalar que la naturaleza de la agresión o violencia indirecta, lleva a que en muchas ocasiones pase desapercibida por parte del profesorado, padres/ madres e incluso por el propio alumnado (Blaya, Debarbieux y Lucas Molina, 2007; Owens y MacMullin, 1995). Además, las víctimas pueden ocultar esta situación por vergüenza o por miedo a las represalias (Baldry y Winkel, 2003; Blaya et al., 2007). Sin embargo, a pesar de ser una forma de agresión más encubierta, las formas de agresión indirecta pueden llegar a tener consecuencias más dañinas y persistentes sobre las víctimas (Owens et al., 2005; Rigby y Slee, 1999). De hecho, Baldry y Winkel (2003) encontraron que las ideaciones suicidas estaban más fuertemente relacionadas con la victimización relacional en la escuela que con la victimización directa. Resultado probablemente motivado por el hecho de que las víctimas se sientan más indefensas y desprotegidas ante la invisibilidad de este tipo de agresión, del que sólo parecen tener constancia ellas y su agresor (que puede incluso llegar a negarlo, atribuyéndolo a una mala interpretación de la víctima).

De todo lo comentado con anterioridad podemos decir que los chicos y las chicas parecen ser vulnerables a distintas clases de victimización como resultado de su interacción con su contexto social más inmediato en la escuela: su grupo de iguales.

Otra variable sociodemográfica que se ha estudiado junto con el género ha sido la edad o el curso. Respecto a esta variable, existe una clara tendencia según la cual el alumnado más pequeño informa sufrir mayores niveles de victimización y agresión que el alumnado de mayor edad (Boulton y Underwood, 1992; Whitney y Smith, 1994). De hecho, estudios de todo el mundo sugieren que existe una fuerte relación inversa entre la edad y las conductas de violencia entre iguales (Olweus, 1999; Owens et al., 2005; Smith et al., 1999b; Sullivan, 2000). La probabilidad de ser víctima de la violencia entre iguales es mucho mayor en los primeros años de Educación Primaria y decrece de forma significativa con el curso (Olweus, 1993); pudiéndose producir un ligero incremento en los primeros años de Educación Secundaria, coincidentes con el período de la preadolescencia (Pellegrini y Long, 2002).

Smith, Madsen y Moody (1999) propusieron dos explicaciones diferentes y complementarias a este decremento con el curso o con la edad: una de tipo evolutiva y otro de tipo socio-contextual. En cuanto a la propuesta evolutiva, el descenso podría venir explicado por una parte, por el hecho de que con la edad el alumnado va 
adquiriendo las habilidades sociales y asertivas necesarias para afrontar de forma efectiva los episodios de violencia, así como para disuadir nuevas agresiones; y por otra, con la edad y los progresos en el desarrollo moral y en la comprensión del mundo social, el alumnado va aprendiendo y entendiendo que no se debe ejercer la agresión sobre otras personas. Respecto a la explicación socio-contextual, se ha hecho referencia por un lado, al hecho de que a menor edad del educando mayor es el porcentaje de alumnado que está en posición de victimizarlo; y por otro, que los educandos más pequeños se encuentran en una situación de menor estatus respecto a los de mayor edad.

En relación a este último enfoque, se encuentra la teoría del Dominio Social propuesta por Pellegrini (2002) para explicar los incrementos en las conductas de violencia entre iguales que tienen lugar al inicio de la Educación Secundaria. Según el autor, este incremento vendría explicado por la renegociación del poder o del estatus que tiene que realizar el alumnado para conseguir una posición social entre sus nuevos pares.

Björqkvist, Lagerspetz y Kaukiainen (1992) comprobaron que, al analizar las diferencias en distintos tipos de violencia, éstas cambiaban de forma significativa con la edad. De este modo, se vio que mientras que las agresiones físicas disminuían con la edad, la violencia verbal se mantenía en una frecuencia constante, y la violencia indirecta o relacional (ignorar, contar mentiras sobre el otro, etc.) aumentaba. La explicación que dieron los autores a estos resultados, a través del desarrollo de su teoría evolutiva de la agresión, fue que con la edad el alumnado va adquiriendo las habilidades verbales y sociales que le permiten pasar de una agresión predominantemente física y primitiva, a un tipo de agresión social, mucho más sofisticada, pasando por la agresión verbal. Aunque algunos estudios más recientes no encuentran estos cambios con la edad (Vaillancourt, Brendgen, Boivin y Tremblay, 2003).

En el presente trabajo se pretende estudiar la relación entre el género y las conductas de violencia entre escolares en Educación Primaria, etapa poco estudiada en nuestro país en el tema que nos atañe, así como examinar posibles diferencias en función de la tipología de agresión y/o victimización. Para ello analizaremos conjuntamente, el efecto que la variable curso tiene en estas posibles diferencias. En concreto, las hipótesis que pretendemos contrastar son: 1) Los chicos sufrirán y ejercerán con mayor frecuencia que las chicas las conductas de agresión física; y 2) Las chicas sufrirán y ejercerán con mayor frecuencia que los chicos las conductas de exclusión y rechazo. Asimismo, buscamos contrastar una tercera hipótesis en relación con el curso: 3) El número de experiencias de violencia entre iguales disminuirá con el curso.

\section{MÉTODO}

\section{Participantes}

En función del objetivo de realizar el estudio en una muestra representativa de la población de alumnado escolarizado en el segundo y tercer ciclo de Educación Primaria de la Comunidad Autónoma de Madrid, se seleccionó a los participantes mediante un muestreo de conglomerados estratificado proporcional al tamaño del centro en una etapa. 
El marco muestral para la selección de la muestra fue la base de centros educativos de la CAM, del que se excluyeron los centros de poblaciones inferiores a 50.000 habitantes.

Para la proporcionalidad se consideró el número de aulas de los centros. Las variables de estratificación fueron la territorialidad de la CAM (centro, norte, sur, este, oeste) y la titularidad del centro (público, privado-concertado). La asignación de los centros a los estratos fue proporcional al de los estratos.

A partir del muestro realizado, se seleccionaron 60 centros de Educación Primaria (30 participantes y 30 suplentes), la selección de los centros suplentes se realizó de acuerdo a las pautas establecidas por organismos nacionales e internacionales en esta materia: el inmediato superior o el inmediato inferior situado en la base de datos.

El número final de centros fue de 27 alcanzando una tasa de respuesta del $90 \%$, tasa relativamente alta.

El número total de participantes, una vez eliminados los cuestionarios defectuosos o incompletos, fue de 2.050 niños y niñas que cursaban segundo y tercer ciclo de Educación Primaria en 27 centros de Educación de Primaria de la Comunidad de Madrid (17 públicos y 10 privados-concertados).

En relación con la distribución en función del género, el porcentaje de chicos fue de un $49,20 \%$ (1.009) y el de chicas de un 50,80\% (1.041). La media de edad para el alumnado participante fue de 9,80 con una desviación típica de 1,24 y un rango de edad entre 8 y 13 años. En cuanto al curso, el 23\% del alumnado estaba escolarizado en $3^{\circ}$, el $25,1 \%$ en $4^{\circ}$, el $26,6 \%$ en $5^{\circ}$ y el $25,4 \%$ en $6^{\circ}$.

\section{Medidas}

Situaciones de victimización por parte de otros/as compañeros/as de clase. Los niveles de victimización informados por el alumnado fueron obtenidos a través de un autoinforme que incluía 20 ítems, con un formato de respuesta tipo Likert de cuatro puntos, sobre distintos episodios de violencia. El alumnado participante podía elegir entre una de las cuatro categorías para describir la frecuencia con la que habían sufrido alguna de las distintas situaciones de victimización durante los dos últimos meses por parte de otros/as compañeros/as de clase: nunca, a veces, bastantes veces, muchas veces. Los 20 elementos fueron sometidos a un análisis de componentes principales seguido de rotación Promax, que permite obtener factores correlacionados. A partir de la orientación proporcionada tanto por el criterio de Kaiser (autovalores mayores que uno), como por el Scree test, así como por consideraciones basadas en la teoría, fueron retenidos dos factores que explicaban el 50,38\% de la varianza total y mostraban una elevada correlación $(r=0,695)$ :

- Victimización física y ataques a la propiedad. Este factor estaba formado por 12 ítems relacionados con situaciones de victimización física (p. ej., me empujan, me pegan) y ataques a la propiedad (p. ej., me roban cosas, me esconden cosas). El conjunto de elementos alcanzaba un coeficiente alpha de 0,91 (IC 95\%: 0,90-0,91), con unos índices de discriminación altos que oscilaban entre 0,47 y 0,72 . 
- Victimización verbal-exclusión social. Formado por 9 elementos que hacían referencia a situaciones de violencia verbal y exclusión social (p. ej., me ignoran, hacen que otros niños no me hablen, me insultan, me ponen motes que no me gustan, etc.). Este factor mostraba una consistencia interna de 0,87 (IC 95\%: 0,86-0,87), los valores de los índices de discriminación oscilaban entre 0,48 y 0,69 .

Situaciones de agresión hacia otros/as compañeros/as de clase. Al igual que para la escala de victimización, los niveles de agresión entre escolares de una misma clase fueron obtenidos con un autoinforme que incluía 20 ítems, con formato de respuesta tipo Likert de 4 puntos, sobre situaciones de agresión. El análisis de componentes principales seguido de rotación Promax, puesto que desde la teoría se esperaba que las dimensiones resultantes estuviesen correlacionadas, reveló la existencia de dos factores bien diferenciados que explicaban el 53,55\% de la varianza total y fuertemente relacionados $(r=0,705)$ :

- Agresión física-ataques a la propiedad. Factor formado por 13 elementos, la mayoría de ellos relacionados con la agresión física y la que se ejerce hacia las propiedades. A excepción de dos ítems: burlarme de su nacionalidad, de su color de piel, etc.; burlarme de su ropa, de sus cosas, etc.; más próximos a las situaciones de rechazo verbal. Aunque en un primer momento se pensó en eliminar estos dos ítems, dado el carácter exploratorio de este estudio se optó finalmente por mantenerlos en el factor. Por una parte, porque se preveía trabajar en los posteriores análisis con las puntuaciones factoriales, de modo que las saturaciones de cada uno de los ítems en los distintos factores quedasen representadas en la estimación de la puntuación mediante el método de la regresión. Y por otro lado, porque interesaba mantener estos ítems en el factor puesto que podrían estar poniendo de manifiesto la particular forma de percibir la agresión por parte del alumnado de estas edades, centrándose en aqueIlo que estaba siendo agredido. En este sentido, la nacionalidad, el color de piel, la ropa, etc., podrían estar siendo considerados como 'propiedades' o atributos del alumnado víctima. Y por consiguiente, los dos ítems burlarme de su nacionalidad, de su color de piel, etc. y burlarme de su ropa, de sus cosas, etc., podrían ser tratados como ejemplos de 'ataques a la propiedad'. El conjunto de los 13 ítems alcanzaba una consistencia interna de 0,92 (IC 95\%: 0,92-0,93). Los índices de discriminación de los ítems mostraban valores entre 0,62 y 0,72.

- Agresión verbal-exclusión social. Este factor estaba formado por 8 elementos, todos ellos relacionados con las situaciones de exclusión social y agresión y rechazo verbal (p. ej., ignorándole, insultándole, poniéndole motes que no le gustan). La consistencia interna de los 8 ítems alcanzaba un valor de 0,87 (IC 95\%: 0,86-0,88). Los índices de discriminación oscilaban entre 0,59 y 0,66.

\section{Procedimiento}

La aplicación fue colectiva y tuvo una duración aproximada de 45 minutos, previa explicación de las instrucciones necesarias y del propósito del estudio, haciendo especial énfasis en la confidencialidad de los datos. 
Se pidió el consentimiento autorizado a todos los padres y madres del alumnado evaluado.

Después del estudio se entregaron informes con los resultados así como pautas de prevención y/o intervención en su caso, a cada uno de los 27 centros participantes.

\section{Resultados}

En el siguiente apartado se presentan los resultados obtenidos sobre las diferencias de género y curso, diferenciando entre las situaciones de victimización y las de agresión.

\section{Situaciones de victimización}

Para poder analizar la relación de las diferentes situaciones de victimización con otras variables, como el género y el curso, se obtuvo una estimación de las puntuaciones del alumnado en cada uno de los factores resultantes de la extracción mediante el método de la regresión. Las puntuaciones factoriales así obtenidas fueron transformadas a puntuaciones típicas de media 50 y desviación típica 10. Se utilizaron estas puntuaciones tipificadas en los dos factores de victimización obtenidos: victimización verbal-exclusión y victimización física-propiedad. Las propiedades psicométricas de estos factores han sido presentadas en el apartado anterior.

En las Tablas 1 y 2 se presentan, respectivamente, los estadísticos descriptivos y el resumen de resultados de los análisis univariantes realizados para la puntuación total y las puntuaciones en los factores de victimización en clase en función del género y del curso.

Tabla 1. Medidas y desviaciones típicas de los factores de victimización por parte de otros compañeros de clase en función del género y del curso.

\begin{tabular}{|c|c|c|c|c|c|c|c|c|}
\hline \multirow{3}{*}{ Género } & \multirow{3}{*}{ Curso } & \multirow{3}{*}{$\mathbf{N}$} & \multicolumn{6}{|c|}{ VICTIMIZACIÓN } \\
\hline & & & \multicolumn{2}{|c|}{ Total } & \multicolumn{2}{|c|}{$\begin{array}{l}\text { Física y ataques } \\
\text { a la propiedad }\end{array}$} & \multicolumn{2}{|c|}{$\begin{array}{c}\text { Verbal y } \\
\text { exclusión social }\end{array}$} \\
\hline & & & Media & $\begin{array}{l}\text { Desv. } \\
\text { tip. }\end{array}$ & Media & $\begin{array}{l}\text { Desv. } \\
\text { tip. }\end{array}$ & Media & $\begin{array}{l}\text { Desv. } \\
\text { tip. }\end{array}$ \\
\hline \multirow[t]{5}{*}{ Chico } & $3^{\mathrm{o}}$ & 230 & 53,65 & 11,43 & 54,29 & 12,07 & 52,12 & 10,52 \\
\hline & $4^{\mathrm{o}}$ & 249 & 51,77 & 11,66 & 51,90 & 11,14 & 51,33 & 11,52 \\
\hline & $5^{\mathrm{o}}$ & 274 & 50,00 & 10,15 & 50,23 & 10,14 & 49,73 & 9,97 \\
\hline & $6^{\mathrm{o}}$ & 256 & 47,85 & 9,05 & 48,02 & 9,00 & 48,05 & 9,12 \\
\hline & Total & 1.009 & 50,72 & 10,78 & 51,01 & 10,82 & 50,24 & 10,40 \\
\hline \multirow[t]{5}{*}{ Chica } & $3^{\mathrm{o}}$ & 241 & 51,38 & 10,04 & 51,48 & 10,07 & 50,93 & 10,05 \\
\hline & $4^{\mathrm{O}}$ & 265 & 50,19 & 9,77 & 50,02 & 9,65 & 50,42 & 10,18 \\
\hline & $5^{\mathrm{o}}$ & 271 & 48,19 & 8,26 & 47,84 & 8,15 & 48,92 & 9,04 \\
\hline & $6^{\mathrm{o}}$ & 264 & 47,64 & 7,94 & 46,99 & 7,51 & 48,90 & 8,99 \\
\hline & Total & 1.041 & 49,30 & 9,13 & 49,02 & 9,03 & 49,76 & 9,60 \\
\hline
\end{tabular}


Según se puede observar en la Tabla 2, los contrastes F univariantes pusieron de relieve diferencias estadísticamente significativas según el curso tanto en la puntuación total como en las dos puntuaciones factoriales. Sin embargo, sólo se encontraron diferencias en función del género en la puntuación total y en el factor de victimización física y ataques a la propiedad. Por otra parte, no se encontraron efectos de interacción en ninguno de los tres casos, tomando el estadístico $F_{(3,2042)}$ los valores $1,03,0,77$ y 1,09 , respectivamente.

Tabla 2. Análisis univariantes de las puntuaciones totales y factoriales en la situación victimización-clase en función del género y del curso.

\begin{tabular}{|c|c|c|c|c|c|c|c|}
\hline \multirow{2}{*}{ VICTIMIZACIÓN } & \multicolumn{4}{|c|}{ Género } & \multicolumn{3}{|c|}{ Curso } \\
\hline & $F_{(1,2042)}$ & $\mathbf{E t a}^{2}$ & $\begin{array}{l}\text { Pot. } \\
\text { Obs. }\end{array}$ & $F_{(3,2042)}$ & $\mathrm{Eta}^{2}$ & $\begin{array}{l}\text { Pot. } \\
\text { Obs. }\end{array}$ & $\begin{array}{l}\text { Games- } \\
\text { Howell }\end{array}$ \\
\hline Total & $11,42^{* *}$ & ,002 & ,992 & $22,63^{* * *}$ & ,032 & 1 & $3^{\circ}, 4^{\circ}>5^{\circ}, 6^{\circ}$ \\
\hline $\begin{array}{l}\text { Física y ataques } \\
\text { a la propiedad }\end{array}$ & $22,08^{* * *}$ & ,011 & ,997 & $28,49 * * *$ &, 040 & 1 & $\begin{array}{c}3^{\circ}>4^{\circ}, 5^{\circ}, 6^{\circ} \\
4^{\circ}>5^{\circ}, 6^{\circ}\end{array}$ \\
\hline $\begin{array}{l}\text { Verbal y } \\
\text { exclusión social }\end{array}$ & 1,37 & ,001 &, 215 & $9,95 * * *$ &, 014 & ,998 & $\begin{array}{c}3^{\mathrm{o}}>5^{\mathrm{o}}, 6^{\mathrm{o}} \\
4^{\mathrm{o}}>6^{\mathrm{o}}\end{array}$ \\
\hline
\end{tabular}

${ }^{*} p<0,05 ;{ }^{* *} p<0,01 ; * * * p<0,001$.

En dicha Tabla también podemos observar que los tamaños del efecto encontrados fueron bastante pequeños.

Examinadas las diferencias entre los cursos por medio del estadístico de GamesHowell se encontraron resultados en la dirección prevista por la hipótesis 3, mostrando que las situaciones de victimización decrecen a medida que el alumnado avanza hacia cursos superiores.

Respecto al género, y también según lo esperado por la hipótesis 1, los chicos revelaron sufrir con mayor frecuencia que las chicas situaciones de victimización física por parte de sus compañeros de clase. En relación con la hipótesis 2, si bien los resultados no van en la dirección esperada, sí podemos observar cómo no existen diferencias entre chicos y chicas en las situaciones de victimización verbal y exclusión social. 


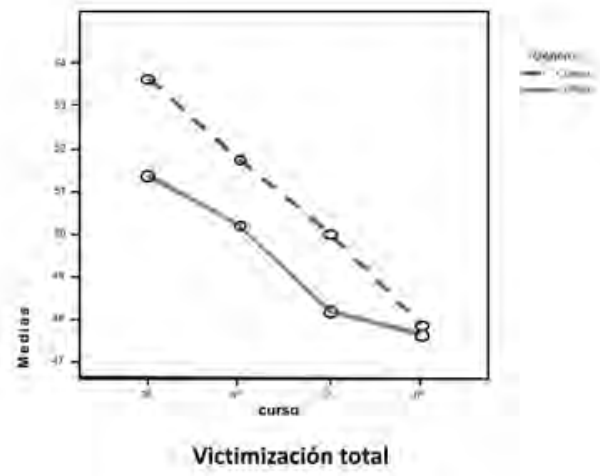

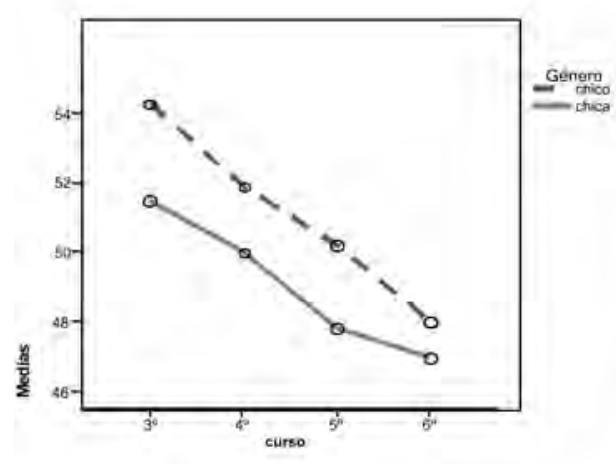

Victimización física y ataques a la propiedad

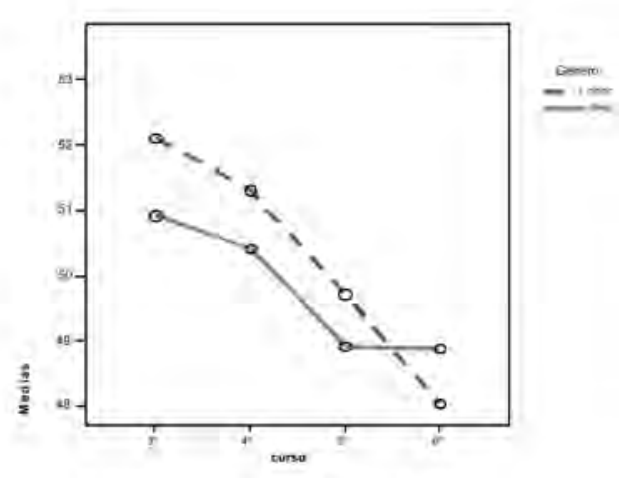

Victimización verbal y exclusión social

Figura 1. Situaciones de victimización sufridas por compañeros de clase en función del género y curso.

\section{Situaciones de agresión}

Al igual que hicimos con las situaciones de victimización, para poder analizar la relación de las diferentes situaciones de agresión con otras variables, como el género y el curso, se utilizaron las puntuaciones factoriales -tipificadas con media 50 y desviación típica 10- y no las diferentes situaciones evaluadas.

En las Tablas 3 y 4 se presentan, respectivamente, los estadísticos descriptivos y el resumen de resultados de los análisis univariantes realizados para la puntuación total y las puntuaciones en los factores de agresión en clase en función del género y del curso. 
Tabla 3. Medias y desviaciones típicas de los factores de agresión hacia compañeros de clase en función del género y del curso.

\begin{tabular}{|c|c|c|c|c|c|c|c|c|}
\hline \multirow{3}{*}{ Género } & \multirow{3}{*}{ Curso } & \multirow{3}{*}{$\mathbf{N}$} & \multicolumn{6}{|c|}{ AGRESIÓN } \\
\hline & & & \multicolumn{2}{|c|}{ Total } & \multicolumn{2}{|c|}{$\begin{array}{l}\text { Física y ataques } \\
\text { a la propiedad }\end{array}$} & \multicolumn{2}{|c|}{$\begin{array}{c}\text { Verbal y } \\
\text { exclusión social }\end{array}$} \\
\hline & & & Media & $\begin{array}{l}\text { Desv. } \\
\text { tip. }\end{array}$ & Media & $\begin{array}{l}\text { Desv. } \\
\text { tip. }\end{array}$ & Media & $\begin{array}{c}\text { Desv. } \\
\text { tip. }\end{array}$ \\
\hline Chico & $3^{\circ}$ & 230 & 52,31 & 12,85 & 52,73 & 13,28 & 51,40 & 12,40 \\
\hline $4^{\circ}$ & 249 & 51,48 & 11,99 & 51,82 & 12,00 & 50,77 & 11,39 & \\
\hline $5^{\mathrm{o}}$ & 274 & 50,79 & 10,41 & 51,03 & 10,57 & 50,27 & 9,88 & \\
\hline $6^{0}$ & 256 & 49,89 & 8,75 & 49,49 & 8,37 & 50,36 & 9,19 & \\
\hline Total & 1.009 & 51,08 & 11,06 & 51,22 & 11,18 & 50,68 & 10,72 & \\
\hline Chica & $3^{\circ}$ & 241 & 48,74 & 10,36 & 48,95 & 9,89 & 48,78 & 10,70 \\
\hline $4^{-0}$ & 265 & 49,08 & 9,11 & 49,51 & 9,77 & 48,73 & 8,42 & \\
\hline $5^{\mathrm{o}}$ & 271 & 48,84 & 8,07 & 48,52 & 7,80 & 49,44 & 8,75 & \\
\hline $6^{\mathrm{O}}$ & 264 & 49,14 & 7,27 & 48,29 & 6,38 & 50,37 & 8,92 & \\
\hline Total & 1.041 & 48,96 & 8,73 & 48,81 & 8,55 & 49,34 & 9,21 & \\
\hline
\end{tabular}

Los contrastes unilaterales revelaron sólo diferencias significativas en función del género en las tres situaciones de agresión en clase. Tal y como predecía nuestra hipótesis 1, los chicos informaron de mayores niveles de agresión física hacia sus iguales que las chicas. Sin embargo, en contra de lo esperado por nuestra hipótesis 2, también informaron ejercer mayores niveles de agresión verbal y exclusión social. De nuevo, los valores de ésta resultaron ser bastante bajos.

Tabla 4. Análisis univariantes de las puntuaciones totales y factoriales en la situación agresión-clase en función del género y del curso.

\begin{tabular}{|c|c|c|c|c|c|c|c|}
\hline \multirow{2}{*}{ AGRESIÓN } & \multicolumn{4}{|c|}{ Género } & \multicolumn{3}{|c|}{ Curso } \\
\hline & $F_{(1,2042)}$ & $\mathbf{E t a}^{2}$ & $\begin{array}{l}\text { Pot. } \\
\text { Obs. }\end{array}$ & $\mathbf{F}_{(3,2042)}$ & $\mathrm{Eta}^{2}$ & $\begin{array}{l}\text { Pot. } \\
\text { Obs. }\end{array}$ & $\begin{array}{l}\text { Games- } \\
\text { Howell }\end{array}$ \\
\hline Total & $24,26^{* *}$ & ,012 &, 998 & 1,06 & ,002 & ,289 & \\
\hline $\begin{array}{l}\text { Física y ataques } \\
\text { a la propiedad }\end{array}$ & $31,31^{* * *}$ & ,015 & 1 & $4,17^{* *}$ & ,006 &, 856 & $3^{\circ}, 4^{\circ}>6^{\circ}$ \\
\hline $\begin{array}{l}\text { Verbal y } \\
\text { exclusión social }\end{array}$ & $9,63^{* *}$ & ,005 & 873 & ,388 & ,001 &, 127 & \\
\hline
\end{tabular}

${ }^{*} \mathrm{p}<0,05 ;{ }^{* *} \mathrm{p}<0,01 ; * * \mathrm{p}<0,00$. 
Únicamente se encontraron diferencias en función del curso en la situación de agresión física y ataques a la propiedad. El estadístico de Games-Howell reveló un descenso de este tipo de agresión en clase a lo largo de los cuatro cursos analizados, tal y como predecía la hipótesis 3 en relación con el curso.

Según se puede observar en los gráficos de medias correspondientes, este decremento con el curso resultó ser mucho más evidente entre los alumnos que entre las alumnas.

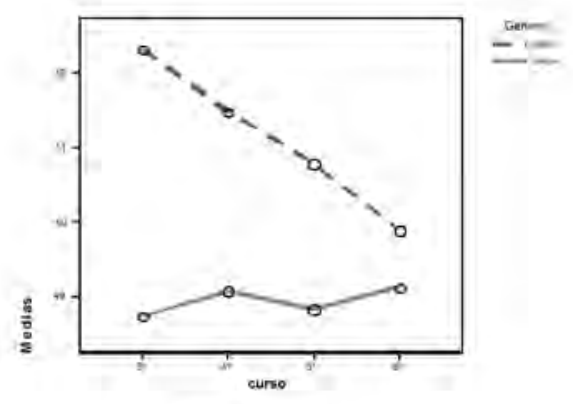

Agresión total

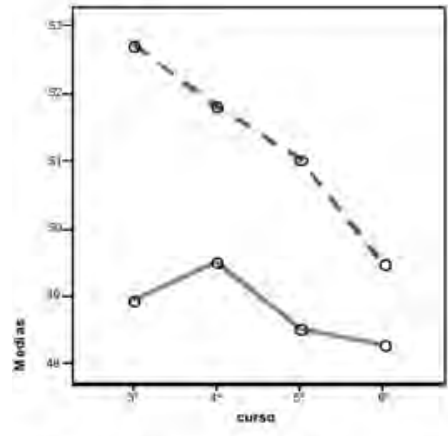

Agresión física y ataques a la propiedad

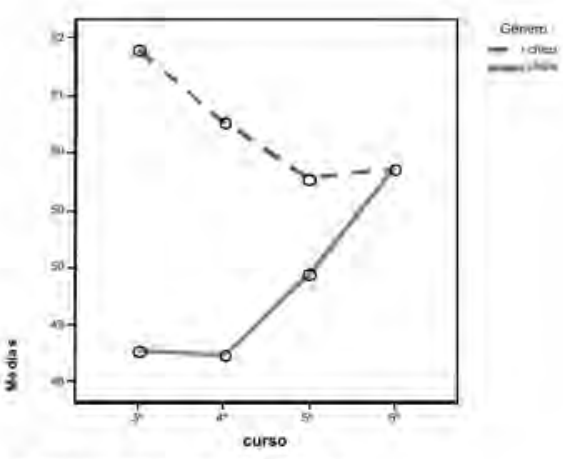

Agresión verbal y exclusión social

Figura 2. Situaciones de agresión ejercidas hacia compañeros de clase en función del género y del curso.

\section{DISCUSIÓN Y CONCLUSIONES}

Respecto a las diferencias de género, los chicos manifestaron sufrir mayor número de situaciones de victimización por parte de sus iguales que las chicas. Estas diferencias resultaron estadísticamente significativas para la victimización física-propiedad y la total. 
En cuanto a las situaciones de agresión, los chicos informaron participar en mayor medida que las chicas; siendo las diferencias estadísticamente significativas para todos los factores analizados (física-propiedad y verbal-exclusión social) y las puntuaciones totales.

Estos resultados que apuntan a una mayor participación de los chicos en las conductas de violencia entre iguales, sobre todo en aquellas modalidades más directas como la violencia física, apoyarían nuestra hipótesis 1 y siguen la línea de lo encontrado en otros estudios, tanto dentro como fuera de España (Akiba et al., 2002; Defensor del Menor, 2006; Defensor del Pueblo, 2000, 2006; Díaz-Aguado et al., 2004; Espelage et al., 2003; Currie et al., 2004; Lucas Molina, 2008; Nansel et al., 2001; Olweus, 1993; Ortega y Mora-Merchán, 2000; Owens et al., 2005; Pellegrini, 2002).

Asimismo, frente a los estudios que señalan una mayor prevalencia de las conductas de exclusión y rechazo social entre las chicas (Crick, 1996; Crick et al., 2001; Crick y Grotpeter, 1995; Martín Seoane et al., 2008) y lo planteado en nuestra hipótesis 2, los resultados de este trabajo irían en la dirección de aquellos que no han encontrado tal diferencia (Nansel et al., 2001; Prinstein et al., 2001; Tapper y Boulton, 2004; Toldos, 2005). Aunque sí es importante señalar que no se detectaron diferencias de género en el número de este tipo de situaciones que informaron sufrir chicos y chicas por parte de sus iguales. Este último resultado puede deberse al hecho de que, en este trabajo, el factor relativo a la exclusión social incluía también aquellas conductas de violencia verbal, que también suelen estar más presentes entre los chicos como destacan otros trabajos (Block, 1983; Knight et al., 2002). Por consiguiente, en futuros estudios sería interesante diferenciar ambos tipos de situaciones, tal vez creando los factores a priori en función del contenido de los ítems, y analizar las posibles diferencias de género a partir de los factores construidos.

Los resultados anteriores en relación con el género ponen de manifiesto el mayor riesgo de violencia que existe entre los chicos probablemente derivado de la asociación de la violencia con valores considerados tradicionalmente masculinos (dominio, fuerza, etc.) y basados en un modelo de relación 'dominio-sumisión' (Cowie, 2000; Díaz-Aguado, 2005). Por consiguiente, plantean la necesidad de adoptar desde las más tempranas edades una perspectiva de género, tanto en la investigación como en la prevención de este tipo de conductas, de forma que se puedan comprender y prevenir las condiciones de riesgo más relevantes tanto para los niños como para las niñas. Como por ejemplo, aquellos programas de prevención que incluyen la lucha contra el sexismo y la violencia en el currículum, con el objetivo de favorecer una construcción de una identidad propia y positiva (DíazAguado y Martínez Arias, 2001). En este sentido, conviene seguir examinando el papel del género en las conductas de violencia entre iguales, así como considerar la participación de las chicas como un importante recurso en la prevención de la violencia escolar.

Respecto a las diferencias en función del curso, se observó un descenso progresivo con el curso en las distintas modalidades de victimización.

Para las situaciones de agresión, aunque también se observó un descenso gradual con el curso -especialmente, entre los chicos-, sólo se encontraron diferencias sig- 
nificativas entre los cursos -más concretamente entre segundo ciclo y $6^{\circ}$ de Primaria- en las conductas de agresión física-propiedad.

Estos resultados siguen la tendencia general encontrada en otros estudios nacionales e internacionales que sugieren una fuerte relación inversa entre el curso o la edad y las conductas de violencia entre iguales (Defensor del Menor, 2006; Defensor del Pueblo, 2000, 2006; Díaz-Aguado et al., 2004; Olweus, 1999; Ortega y Mora-Merchán, 2000; Owens et al., 2005; Síndic de Greuges, 2007; Smith et al., 1999b; Sullivan, 2000; Whitney y Smith, 1994) y apoyarían nuestra hipótesis 3. Para explicarlos podemos considerar las dos hipótesis planteadas por Smith et al. (1999a): la evolutiva y la socio-contextual. Desde el planteamiento evolutivo, el decremento en las conductas de victimización y de agresión física-propiedad, podría explicarse por el hecho de que con la edad el alumnado va siendo socializado bajo la idea de que no está bien agredir a otras personas, así como va adquiriendo las habilidades sociales y asertivas necesarias para hacer frente de forma efectiva a las situaciones de victimización. Desde la hipótesis socio-contextual, el alumnado, a medida que avanza en el curso, tiene menos riesgo de ser agredido por escolares de mayor edad, así como va adquiriendo mayor estatus por lo que ve reducida su probabilidad de ser victimizado. En este sentido, conviene señalar el papel que puede tener el alumnado de mayor edad en la prevención e intervención de los episodios de violencia entre iguales. Por otra parte, conviene seguir avanzando en el estudio de esta variable, incluyendo el posible efecto de otros factores (como por ejemplo, el estatus del alumnado entre sus iguales) de forma que podamos comparar el poder explicativo de la perspectiva evolutiva y la socio-contextual.

Finalmente, creemos necesario mencionar algunas limitaciones del presente estudio. En primer lugar, señalar el hecho de haber empleado como técnica de recogida de datos el autoinforme, con los sesgos de deseabilidad social que ello supone. Limitación que convendría solventar en futuras investigaciones adoptando una metodología multi-informante e incluyendo a otros participantes de la comunidad educativa como profesorado, padres y madres, etc. Por otra parte, también sería interesante completar la metodología cuantitativa con el uso de otra de carácter cualitativo (como por ejemplo, a través de entrevistas individuales, grupos de discusión, etc.).

\section{REFERENCIAS BIBLIOGRÁFICAS}

ARARTEKO (2006). Convivencia y conflictos en los centros educativos. Vitoria: Ararteko.

BALDRY, A. C. y WINKEL, F. W. (2003). Direct and vicarious victimization at school and at home as risk factors for suicidal cognition among Italian adolescents. Journal of Adolescence, 26, 703-716.

BLAYA, C., DEBARBIEUX, E. y LUCAS MOLINA, B. (2007). La violencia hacia las mujeres y hacia otras personas percibidas como distintas a la norma dominante: el caso de los centros educativos. Revista de Educación, 342, 61-81.

BJÖRKQVIST, D., LAGERSPETZ, K. y KAUKIAINEN, A. (1992). Do girls manipulate and boys fight? Developmental trends in regard to direct and indirect aggression. Aggressive Behavior, 18, 117-127. 
BLOCK, J. H. (1983). Differential premises arising from differential socialization of the sexes: Some conjectures. Child Development, 54, 1.335-1.354.

BOULTON, M. y UNDERWOOD, K. (1992). Bully/victim problems among middle school children. British Journal of Educational Psychology, 62, 73-87.

COWIE, H. (2000). Bystanding or standing by: Gender issues in coping with bullying in schools. Aggressive Behavior, 26, 85-97.

CRICK, N. R. (1996). The role of overt aggression, relational aggression, and prosocial behavior in the prediction of children's future social adjustment. Child Development, 67, 2.317-2.327.

CRICK, N. R. y GROTPETER, J. K. (1995). Relational aggression, gender, and socialpsychological adjustment. Child Development, 66, 710-722.

CRICK, N. R. y GROTPETER, J. K. (1996). Children's treatment by peers: Victims of relational and overt aggression. Development and Psychopathology, 8, 367-380.

CRICK, N. R., NELSON, D. A., MORALES, J. R., CULLERTON-SEN, S. y HICKMAN, S. E. (2001). Relational aggression in childhood and adolescence. En J. Juvonen y S. Graham (Eds.), Peer harassment in school: The plight of the vulnerable and victimized, pp. 196-214. New York: Guilford.

CURRIE, C., ROBERTS, C., MORGAN, A., SMITH, R., SETTERTOBULTE, W., SAMDAL, O. y BARNEKOW, V. (Eds.) (2004). Young people's health in context. Health Behaviour in School-aged Children (HSBC) study. Copenhague: WHO Publications

DEFENSOR DEL PUEBLO (2000). Violencia escolar: el maltrato entre iguales en la Educación Secundaria Obligatoria. Madrid: Publicaciones del Defensor del Pueblo.

DEFENSOR DEL PUEBLO (2006). Violencia escolar: el maltrato entre iguales en la Educación Secundaria Obligatoria (1999-2006). Madrid: Publicaciones del Defensor del Pueblo.

DEFENSOR DEL MENOR de la Comunidad de Madrid (2006). Convivencia y confrontación entre iguales. Madrid: Publicaciones del Defensor del Menor de la Comunidad de Madrid.

DÍAZ-AGUADO, M. J. (2005). La violencia entre iguales en la adolescencia y su prevención desde la escuela. Psicothema, 17, 549-558.

DÍAZ-AGUADO, M. J., MARTÍNEZ ARIAS, R. y MARTÍN SEOANE, G. (2004). Prevención de la violencia y lucha contra la exclusión desde la adolescencia. Volumen uno: La violencia entre iguales en la escuela y en el ocio: estudios comparativos e instrumentos de evaluación. Madrid: INJUVE.

ESPELAGE, D., BOSWORTH, D. y SIMON, T. (2000). Examining the social context of bullying behaviours in early adolescence. Journal of Counselling and Development, 78, 326-333.

ESPELAGE, D., HOLT, M. K. y HENKEL, R. R. (2003). Examination of peer-group contextual effects on aggression during early adolescence. Child Development, 74, 205-220.

GARAIGORDOBIL, M. y OÑEDERRA, J. A. (2009). Acoso y violencia escolar en la comunidad autónoma del País Vasco. Psicothema, 21, 83-89.

KNIGHT, G. P., GUTHRIE, I. K., PAGE, M. C. y FABES, R. A. (2002). Emotional arousal and gender differences in aggression. En J. Juvonen y S. Graham (Eds.), Peer 
harassment in school: The plight of the vulnerable and victimized, pp. 25-72. New York, NY: Guilford.

LUCAS MOLINA, B. (2008). Violencia escolar en Educación Primaria. Tesis Doctoral Inédita. Madrid: Universidad Complutense.

MARTíN SEOANE, G., PULIDO, R. y VERA, R. (2008). Maltrato entre iguales y exclusión social en la Comunidad de Madrid: análisis y posibilidades de intervención. Psicología Educativa, 14, 103-113.

NANSEL, T. R., OVERPECK, M., PILLA, R. S., RUAN, W. J., SIMONS-MORTON, B. y SCHEIDT, P. (2001). Bullying behavior among US youth: Prevalence and association with psychosocial adjustment. The Journal of the American Medical Association, 285, 2.094-2.100.

NANSEL, T., CRAIG, W., OVERPECK, M. , SALUJA, G., RUAN, W. y the Health Behavior in School-Aged Children Bullying Analyses Working Group. (2004). Cross-national consistency in the relationship between bullying behaviors and psychosocial adjustment. Archives of Pediatrics y Adolescent Medicine, 158, 730-736.

OLWEUS, D. (1978). Aggression in the schools: bullies and whipping boys. Washington D. C.: Hemisphere (Wiley).

OLWEUS, D. (1993). Conductas de acoso y amenaza entre escolares. Madrid: Morata. (1998 fecha de la edición en castellano).

OLWEUS, D. (1999). Norway. En Smith, P.K., Morita, Y., Junger-Tas, J., Olweus, D., Catalano, R., y Slee, P. (Eds). The nature of school bullying. A cross-national perspective. London: Routledge.

ORTEGA, R. y MORA-MERCHÁN, J. A. (2000). Violencia escolar: mito o realidad. Sevilla: Mergablum.

OWENS, L. D. y MACMULLIN, C. E. (1995). Gender differences in aggression in children and adolescents in South Australian schools. International Journal of Adolescence and Youth, 6, 21-35.

OWENS, L., DALI, A. y SLEE, P. (2005). Sex and age differences in victimisation and conflict resolution among adolescents in a South Australian school. Aggressive Behavior, 31, 1-12.

PARKE, R. D. y SLABY, R. G. (1983). The development of aggression. In P. MUSEN (Series Ed.) y E. M. HETHERINGTON (Ed.), Handbook of child psychology: Vol. 4. Socialization, personality, and social development, pp. 547-641. New York: Wiley.

PELLEGRINI, A. D. (2002). Bullying and victimization in middle school: A dominance relations perspective. Educational Psychologist, 37, 151-163.

PELLEGRINI, A. D. y LONG, J. (2002). A longitudinal study of bullying, dominance, and victimization during the transition from primary to secondary school. British Journal of Developmental Psychology, 20, 259-280.

PRINSTEIN, M. J., BOERGERS, J. y VERNBERG, E. M. (2001). Overt and relational aggression in adolescents: Social-psychological adjustment of aggressors and victims. Journal of Clinical Child Psychology, 30, 479-491. 
RIGBY, K. y SLEE, P. T. (1999). Suicidal ideation among adolescent school children, involvement in bully/victim problems and perceived low social support. Suicide and Life-threatening Behavior, 29, 119-130.

SALMIVALLI, C. (2010). Bullying and the peer group: A review. Aggression and Violent Behavior, 15, 112-120.

SMITH, P. K., MADSEN, K. C. y MOODY, J. C. (1999a). What causes the age decline in reports of school being bullied at school? Toward a developmental analysis of risks of being bullied. Educational Research, 41, 267-285.

SMITH, P. K., MORITA, Y., JUNGER-TAS, J., OLWEUS, D., CATALANO, R. y SLEE, P. (Eds). (1999b). The nature of school bullying. A cross-national perspective. London: Routledge.

SMITH, P. K. y SHARP, S. (Eds.) (1994). School Bullying. Insights and perspectives. New York: Routledge.

SULLIVAN, K. (2000). Planning, philosophy, and policy. En K. Sullivan (Ed.), The anti-bullying handbook, pp. 9-39. New York: Oxford University Press.

TAPPER, K. y BOULTON, M. (2004). Sex differences in levels of physical, verbal, and indirect aggression amongst primary school children and their associations with beliefs about aggression. Aggressive Behavior, 30, 123-145.

TOLDOS, M. P. (2005). Sex and age differences in self-estimated physical, verbal and indirect aggression in Spanish adolescents. Aggressive Behavior, 31, 13-23.

VAILLANCOURT, T., BRENDGEN, M., BOIVIN, M. y TREMBLAY, R. E. (2003). A longitudinal confirmatory factor analysis of indirect and physical aggression: Evidence of two factors over time? Child Development, 74, 1628-1638.

VEENSTRA, R., LINDENBERG, S., MUNNIKSMA, A. y DIJKSTRA, J. (2010). The complex relation between bullying, victimization, acceptance, and rejection: Giving special attention to status, affection, and sex differences. Child Development, 81, 510-516.

WHITNEY, I. y SMITH, P. K. (1993). A survey of the nature and extend of bullying in junior/middle and secondary schools. Educational Research, 35, 3-25. 